\title{
Expression profile of Twist, vascular endothelial growth factor and CD34 in patients with different phases of osteosarcoma
}

\author{
PENGFEI LEI, DENGFENG DING, JIE XIE, LONG WANG, QIANDE LIAO and YIHE HU \\ Department of Orthopedics, Xiangya Hospital of Central South University, Changsha, Hunan 410008, P.R. China
}

Received August 18,2014; Accepted April 29, 2015

DOI: $10.3892 / 01.2015 .3246$

\begin{abstract}
The aim of the present study was to investigate the clinical significance of Twist, vascular endothelial growth factor (VEGF) and CD34 expression in osteosarcoma (OS) in order to elucidate potential therapeutic targets for the treatment of OS. Immunohistochemistry was performed to detect the protein expression of Twist, VEGF and CD34 in OS and osteochondroma (OC) tissues. The ratio of the protein expression of Twist and VEGF in OS and OC tissues as well as at different phases of OS was compared using chi-squared tests. Microvessel density (MVD), as determined by CD34 labeling, in OS and OC tissue as well as at different phases of OS was compared using the Student's t-test. In addition, associations between Twist, VEGF and MVD were assessed using the Spearman's rank correlation test. The results revealed that out of the 32 OS tissues examined, $56.25 \%$ exhibited Twist positive expression, $71.88 \%$ exhibited VEGF positive expression and the MVD was increased compared with that of the OC tissue. The positive rate of Twist and VEGF expression in phase III OS tissues was significantly increased compared with that in phase I/II OS tissues (Twist: $\chi^{2}=5.732$, $\mathrm{P}=0.018$; VEGF: $\left.\chi^{2}=7.513, \mathrm{P}=0.006\right)$. The MVD in phase III OS tissues (31.08 \pm 3.36 per field) was significantly higher compared with that of the phase I/II OS tissues (41.2 \pm 4.17 per field; $t=7.536, \mathrm{P}<0.001)$. Spearman's rank correlation analysis revealed that Twist expression was positively associated with VEGF expression ( $\mathrm{r}=0.371, \mathrm{P}=0.002)$ and with MVD $(\mathrm{r}=0.393$, $\mathrm{P}=0.001)$ in $\mathrm{OS}$; in addition, VEGF expression was found to have a positive correlation with MVD $(r=0.469, \mathrm{P}=0.001)$. In conclusion, the results of the present study demonstrated that OS tissues exhibited elevated Twist and VEGF expression as well as MVD compared with OC tissue. In addition, metastatic OS (phase III) exhibited an increased positive rate of Twist and VEGF expression as well as MVD values compared with
\end{abstract}

Correspondence to: Professor Yihe Hu, Department of Orthopedics, Xiangya Hospital of Central South University, 87 Xiangya Road, Changsha, Hunan 410008, P.R. China

E-mail: csuhuyihe@163.com

Key words: Twist, vascular endothelial growth factor, CD34, osteosarcoma, metastatic non-metastatic OS (phase I/II). Furthermore associations were detected between Twist and VEGF expression as well as VEGF and MVD. Therefore, inhibition of Twist expression may have potential therapeutic use for the treatment of OS.

\section{Introduction}

Osteosarcoma (OS) is one of the most prevalent types of malignant bone tumors, which predominantly occurs in adolescents and young adults; in addition, OS has a morbidity rate of $\sim 5$ cases per million (1). The conventional treatment of OS consists of surgery and radiotherapy; however, additional radiotherapy may not be used due to the risk of radiation-induced necrosis of surrounding structures (2). In addition, there is a high risk of relapse or metastasis for OS patients, even following curative resection; therefore, a substantial portion of patients with OS respond poorly to chemotherapy (3). Thus, elucidating the mechanisms underlying the metastasis of OS may lead to the development of novel therapeutic strategies for the treatment of OS.

Twist is a member of a highly conserved basic helix-loop-helix transcription factor family and is located on human chromosome 7 (4). It has been reported that Twist mediates cell migration and differentiation under various physiological conditions (5). In addition, Twist has been demonstrated to significantly enhance tumor malignancies, including breast cancer (6), hepatocellular carcinoma (HCC) (7) and prostate cancer (8). It was reported that the role of Twist in tumor cell invasion and metastasis may be associated with the regulation of cancer-associated functions, such as angiogenesis (9). Furthermore, Twist was found to regulate apoptosis and angiogenesis under a variety of pathological conditions (10). Therefore, it was hypothesized that Twist was closely correlated with malignant potential, progression and survival in patients with OS. However, to the best of our knowledge, there is limited information regarding the pathological roles of Twist expression in human OS tissues.

Angiogenesis is a key factor that mediates tumor metastasis; in addition, correlations between angiogenesis and patient survival in OS have been previously reported (11). Microvessel density (MVD) was demonstrated to be increased in histopathologically aggressive cancers, including esophageal squamous cell carcinoma (12) and mammary carcinoma (13). High MVD was also reported to be correlated with metastasis and poor survival in various types of cancer, such as OS (14). Increasing evidence has suggested that MVD may be considered an 
indirect marker of neoangiogenesis, which is commonly labeled by CD31 or CD34 (14).

Vascular endothelial growth factor (VEGF), the most important mediator of vascular formation, is essential for the initiation of immature vessel formation (15). VEGF has been reported to be directly involved in the angiogenesis process, tumor growth and metastasis (16). Neovascularization, promoted by VEGF, is known to reflect the aggressiveness and the metastatic potential of OS (17). However, current understanding of the correlation of Twist, VEGF and MVD in human OS tissues is limited.

The present study aimed to investigate Twist, VEGFandCD34 expression levels in OS tissues, using immunohistochemical (IHC) staining, in order to illustrate correlations among these components in OS.

\section{Materials and methods}

Tissues. A total of 32 cases of different phase OS and 10 cases of osteochondroma (OC), a benign tumor of the bone, were obtained from patients who underwent surgery between June 2011 and March 2013 at the Department of Orthopedics, Xiangya Hospital of Central South University (Changsha, China). Patients with OS underwent amputation surgery, while patients with OC underwent curettage. All OS tumor tissues were formalin-fixed and paraffin-embedded following resection and then pathologically diagnosed (18): Phase I OS, 3 cases; phase II OS, 17 cases; and phase III OS, 12 cases. Ages of patients range from 9 to 54 years old (mean, 23.21 \pm 8.73 ), including 18 males and 14 females. In 20 cases OS was identified in the femur, in 8 cases the malignancy was located in the tibia and the remaining 4 cases were located in other bone regions. OC tissues were used as the control. All tissues were collected prior to any chemoradiotherapy. Written informed consent was obtained from the patients. The study was approved by the Ethics Committee of the Xiangya Hospital of Central South University.

Immunohistochemical staining. Immunohistochemical staining was performed using the streptavidin-peroxidase (SP) method (SP kit; ZSGB-Bio, Beijing, China), according to the manufacturer's instructions. Slides were deparaffinized with xylene (BaiYi, Co. Ltd., Jining, China) twice for $30 \mathrm{~min}$ each, dehydrated three times in a gradient series of ethanol (100, 95, 90, 80 and $70 \%$ ) and rinsed with phosphate-buffered saline (PBS). Following 15 min of treatment with $3 \% \mathrm{H}_{2} \mathrm{O}_{2}$, slides were blocked using normal goat serum (Jackson ImmunoResearch, West Grove, PA, USA) for $20 \mathrm{~min}$. Slides were incubated with the following primary antibodies overnight at $4^{\circ} \mathrm{C}$ : Rabbit polyclonal anti-Twist (1:50; cat. no. ab50581; Abcam, Cambridge, UK), mouse monoclonal anti-VEGF (1:150; cat. no. ab1316; Abcam) or mouse monoclonal anti-CD34 (1:100; cat. no. ab8536; Abcam). Slides were subsequently washed three times with PBS for $15 \mathrm{~min}$. Slides were treated with SP reagent for $20 \mathrm{~min}$ and then incubated with a secondary antibody for $90 \mathrm{~min}$ at $37^{\circ} \mathrm{C}$, using an SP rabbit and mouse horseradish peroxidase kit (cat. no. CW0120; CWBiotech Co. Ltd., Wuhan, China) according to the manufacturer's instructions. Subsequently, the slides were washed twice with PBS for 15 min per wash, and visualized using 3,3'-diaminobenzidine for $5 \mathrm{~min}$ and then counterstained
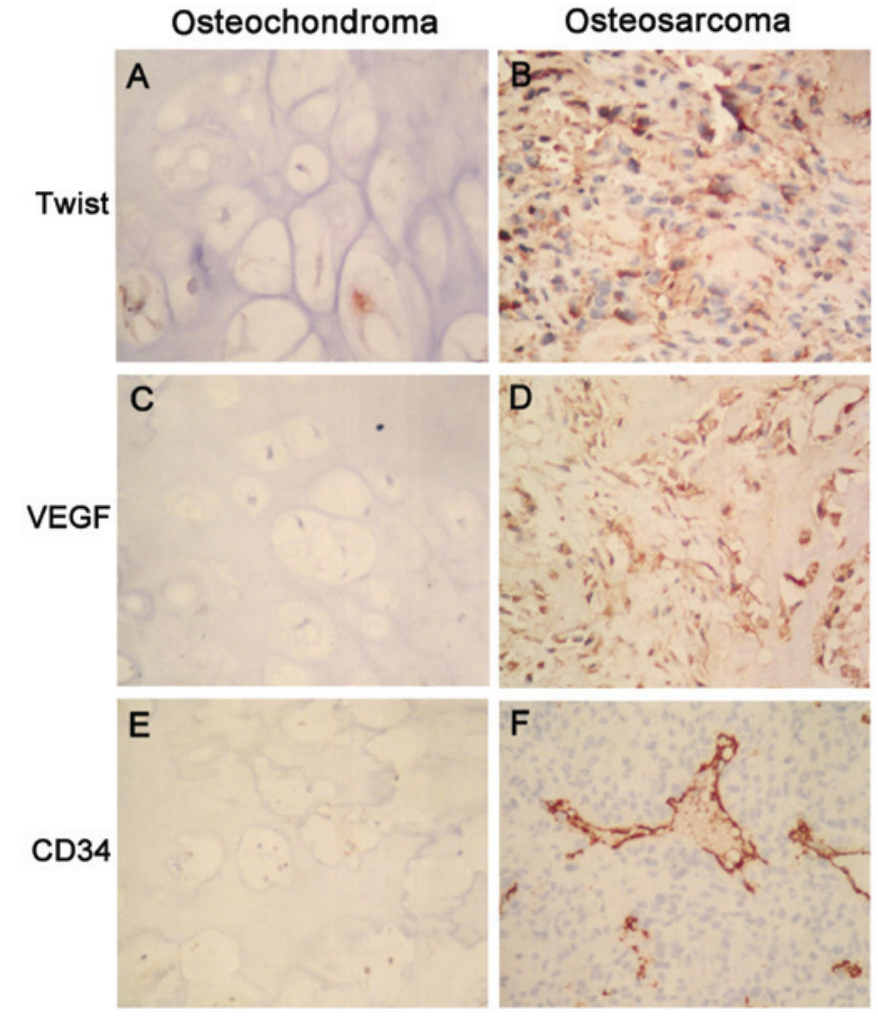

Figure 1. Representative images of immunohistochemical staining for Twist, VEGF and CD34 protein expression. Twist expression in (A) osteosarcoma and (B) osteochondroma tissues; VEGF expression in (C) osteosarcoma and (D) osteochondroma tissues; and CD34 expression in (E) osteosarcoma and (F) osteochondroma tissues. Magnification, x400.

with hematoxylin (Solarbio Science \& Technology Co., Ltd., Beijing, China). The slides were mounted and dried. Images were captured using an Olympus microscope (C-7070; Olympus Corporation, Tokyo, Japan).

Evaluation of staining. Slides were evaluated by two investigators, who were blinded to experiments, under a light microscope (BX43; Olympus Corporation). Twist and VEGF staining intensity were scored as follows: 0 , negative; 1 , weak; 2 , medium; and 3, strong. Extent of staining was scored as: 0, $0 \% ; 1,1-25 \% ; 2,26-50 \% ; 3,51-75 \%$; and $4,76-100 \%$. The final staining score (0-7) was calculated as the sum of the intensity score and extent score. Staining scores of 0-1 were considered to be negative, scores of 2-3 were considered as low expression and score of $>3$ were considered as high expression.

Measurement of MVD. Slides were examined at low-power magnification (x40; microscope, BX43) to identify the areas with the highest density of microvessels (labeled by CD34). In each case, the most vascularized area was selected and the microvessels within a high-power magnification (x200) field of vision were counted three times. Macrovascular structures with smooth muscle cells were excluded. The mean of the three highest counts per tumor was used for analysis.

Statistical analysis. SPSS 16.0 software (SPSS, Inc., Chicago, IL, USA) was used to perform statistical analysis. The ratio of high expression of Twist and VEGF in different phases of OS was compared using chi-squared tests. MVD in different 
Table I. Twist expression in osteochondroma and osteosarcoma.

\begin{tabular}{|c|c|c|c|c|c|c|}
\hline Tumor & Cases & Negative cases & Positive cases & Positive rate, $\%$ & $\chi^{2}$ & P-value \\
\hline Osteochondroma & 10 & 9 & 1 & 10.00 & \multirow{2}{*}{6.579} & \multirow{2}{*}{0.01} \\
\hline Osteosarcoma & 32 & 14 & 18 & 56.25 & & \\
\hline
\end{tabular}

Table II. Vascular endothelial growth factor expression in osteochondroma and osteosarcoma.

\begin{tabular}{lccccc}
\hline Tumor & Cases & Negative cases & Positive cases & Positive rate, $\%$ & $\chi^{2}$ \\
\hline Osteochondroma & 10 & 8 & 2 & 20.00 & 8.510 \\
Osteosarcoma & 32 & 9 & 23 & 71.88 & 0.004 \\
\hline
\end{tabular}

Table III. MVD values in osteochondroma and osteosarcoma.

\begin{tabular}{lcrcc}
\hline Tumor & Cases & MVD $^{\mathrm{a}}$ & $\chi^{2}$ & P-value \\
\hline $\begin{array}{l}\text { Osteochondroma } \\
\text { Osteosarcoma }\end{array}$ & 10 & $7.97 \pm 3.67$ & 17.086 & 0.008 \\
\hline
\end{tabular}

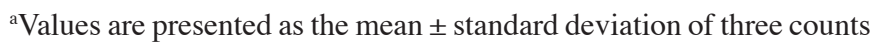
of MVD within a high-power magnification (x200) field. MVD, microvessel density.

phases of OS was compared using Student's t-tests. Associations among Twist, VEGF and MVD were assessed using the Spearman's rank correlation test. $\mathrm{P}<0.05$ was considered to indicate a statistically significant difference between values.

\section{Results}

Expression of Twist, VEGF and CD34 in OS and OC tissues. Immunohistochemical staining data revealed that Twist was located in the nuclei and cytoplasm, VEGF was identified in the cytoplasm and CD34 (for MVD) in the microvessels (Fig. 1A-F). The positive expression of Twist was detected in 18 of the total 32 OS tissues, but in only 1 of the 10 OC tissues; therefore, the positive rate of Twist in OS tissues was significantly increased compared with that in OC tissues $\left(\chi^{2}=6.579\right.$, $\mathrm{P}=0.01$ ) (Table I). In addition, the positive expression of VEGF was detected in 23 out of 32 OS tissues and in only 2 of the $10 \mathrm{OC}$ tissues, demonstrating that the positive rate of VEGF in OS tissues was significantly higher compared with that of OC tissues $\left(\chi^{2}=8.510, P=0.004\right)$ (Table II). Furthermore, MVD in OS tissues was significantly elevated compared with that in OC tissues $(\mathrm{t}=17.086, \mathrm{P}=0.008)$ (Table III).

Expression of Twist, VEGF and CD34 in different phases of $O S$. The positive expression of Twist was detected in 10 of the total 12 phase III OS tissues and in 8 of the 20 phase I/II OS tissues; therefore, the positive rate of Twist in phase III OS was significantly higher compared with that in phase I/II OS $\left(\chi^{2}=5.732, \mathrm{P}=0.018\right)$ (Table IV). The positive expression of VEGF was detected in all 12 phase III OS tissues and in 11 out of 20 phase I/II OS tissues; thus, the positive rate of VEGF in phase III OS was significantly increased compared with that in phase I/II OS $\left(\chi^{2}=7.513, \mathrm{P}=0.006\right)$ (Table V). The MVD in phase III OS tissues $(41.2 \pm 4.17$ per field) was significantly increased compared with that of phase I/II OS tissues $(31.08 \pm 3.36$ per field) $(\mathrm{t}=7.536, \mathrm{P}<0.001)$ (Table VI).

Associations among Twist, VEGF and MVD expression in correlation analysis. Spearman's rank correlation analysis revealed that Twist expression was positively associated with VEGF expression $(r=0.371, P=0.002)$ and with MVD $(r=0.393$, $\mathrm{P}=0.001)$ in $\mathrm{OS}$; in addition, VEGF expression was demonstrated to have a positive correlation with MVD $(\mathrm{r}=0.469, \mathrm{P}=0.001)$.

\section{Discussion}

OS is an aggressive type of cancer that affects the skeletal system. Although investigated by numerous previous studies, the molecular mechanisms of the etiology and pathogenesis underlying OS remain to be elucidated $(19,20)$. Therefore developing effective therapeutic strategies for the treatment of OS is challenging. The present study demonstrated that Twist expression was positively correlated with tumor phase and metastasis in patients with OS. It has previously been reported that Twist expression was significantly higher in cancer cells of sarcomatoid renal cell carcinoma compared with those at the edge of the tumors; in addition, Twist was associated with tumor aggressiveness and poor prognosis in patients with renal cell carcinoma (21). Oncogenic activation of Twist was reported to be essential for the epithelial-mesenchymal transition (EMT) that initializes invasion and metastasis (22). Twist has been demonstrated to couple aberrant signals from EMT to senescence and was found to be an important candidate biomarker for cervical cancer prognosis (23). In addition, the downregulation of Twist expression was suggested to facilitate apoptosis and recover the sensitivity of chemoresistance induced by cisplatin in ovarian cancer (24). Furthermore, the enhanced production of Twist resulted in VEGF secretion that promotes tumor angiogenesis in breast cancer cells, which in turn enhances cancer invasion and metastasis (9).

VEGF, as a prime mediator of angiogenesis, has been implicated in carcinogenesis and metastasis in human and murine OS cells (25). It was reported that the survival and proliferation of highly aggressive OS cells was dependent on autocrine 
Table IV. Twist expression in different phase of osteosarcoma.

\begin{tabular}{lrrrrr}
\hline Phase & Cases & Negative cases & Positive cases & Positive rate, $\%$ & $\chi^{2}$ \\
\hline I & 3 & 3 & 0 & 0.00 & P-value \\
II & 17 & 9 & 8 & 47.06 & $5.732^{\mathrm{a}}$ \\
III & 12 & 2 & 10 & 83.33 & $0.018^{\mathrm{a}}$ \\
\hline
\end{tabular}

aPhase I and II vs. phase III.

Table V. Vascular endothelial growth factor expression in different phases of osteosarcoma.

\begin{tabular}{lcccrr}
\hline Phase & Cases & Negative cases & Positive cases & Positive rate, $\%$ & $\chi^{2}$ \\
\hline I & 3 & 3 & 0 & 0.00 & \\
II & 17 & 6 & 11 & 64.71 & $7.513^{\mathrm{a}}$ \\
III & 12 & 0 & 12 & 100.00 & $0.006^{\mathrm{a}}$ \\
\hline
\end{tabular}

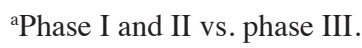

Table VI. MVD values in different phase of osteosarcoma.

\begin{tabular}{lccc}
\hline Phase & Cases & MVD $^{\mathrm{a}}$ & t-value \\
\hline I & 3 & $23.33 \pm 4.36$ & \\
II & 17 & $37.15 \pm 3.74$ & $7.536^{\mathrm{b}}$ \\
I and II & 20 & $31.08 \pm 3.36$ & $<0.0001^{\mathrm{b}}$ \\
III & 12 & $41.20 \pm 4.17$ & \\
\hline
\end{tabular}

${ }^{a}$ Values are presented as the mean \pm standard deviation of three counts of MVD within a high-power magnification (x200) field; ${ }^{b}$ phase I and II vs. phase III. MVD, microvessel density.

VEGF/VEGF receptor 1 signaling in vitro (17). Numerous studies have suggested that VEGF expression may act as a biomarker of prognosis in OS patients (26-28). A previous study reported that patients with high VEGF expression had significantly reduced disease-free survival and overall survival rates compared with OS patients with low or negative VEGF expression (28). In line with previous studies, the present study determined that the positive rate of VEGF in OS tissues was significantly increased compared with that in OC tissues with increased grade and metastasis, suggesting that VEGF expression may be an efficient biomarker for predicting the prognosis of OS patients. However, further large-scale prospective trials are required in order to confirm the prognostic value of VEGF for survival in OS patients.

The present study determined that Twist expression was positively correlated with VEGF expression in OS. Niu et al (7) reported that increased Twist messenger RNA and protein expression levels were positively associated with the upregulation of VEGF expression in HCC patients with a poor prognosis, suggesting that Twist may have a critical role in the angiogenesis and metastasis of HCC (7). In cases of supraglottic carcinoma, Twist and VEGF expression levels in lymph node metastasis patients were significantly increased compared with those in patients without metastasis; in addition, the levels of VEGF were reported to be positively correlated with those of Twist (29). One study reported that stable overexpression of Twist in the MCF-7 breast cancer cell line resulted in increased VEGF synthesis in vitro and xenograft experiments with MCF-7 cells overexpressing Twist produced tumors with a higher vascular volume and vascular permeability in vivo (6). Overall, these previous studies indicated that Twist overexpression may enhance cancer invasion and metastasis through increasing VEGF expression, resulting in the induction of angiogenesis, which is pivotal for the transformation into an aggressive phenotype.

In the present study, the staining of endothelial cells for CD34 was used to evaluate the MVD in OS tissues. The results indicated that the MVD in OS tissues was significantly increased compared with that of OC tissues; in addition, this increase was correlated with OS phase. Spearman's rank correlation analysis revealed that Twist expression was positively correlated with MVD, while VEGF expression also exhibited a positive correlation with MVD. Positive Twist expression in HCC specimens was previously demonstrated to have an elevated MVD compared with specimens with negative Twist expression (7). Another study demonstrated that the MVD in paraffin sections from 97 patients with HCC was correlated with the upregulation 
of Twist expression (30). In addition, Twist expression was reported to be positively associated with MVD in cancer cells of sarcomatoid renal cell carcinoma (21). It has been demonstrated that VEGF regulated the development of microvessels and was significantly correlated with MVD in Ewing's sarcoma family of tumors (31). Serum VEGF levels were identified to be significantly elevated in OS patients with pulmonary metastasis compared with patients without detectable disease relapse; in addition, these VEGF levels were positively correlated with the MVD, suggesting that the pre-therapeutic serum VEGF levels reflected the angiogenic property of OS $(32,33)$. However, Ek et al (14) demonstrated that the degree of MVD and VEGF expression did not provide prognostic information for OS, as determined through a small-scale (25 cases) investigation. Thus, the association between VEGF expression and MVD in OS requires further large-scale investigations, in addition to further studies regarding Twist and VEGF expression.

In conclusion, the present study investigated the expression of Twist, VEGF and CD34 (MVD) in 32 cases of OS at different phases and analyzed the associations among Twist, VEGF and MVD. The results revealed that OS tissues exhibited elevated expression levels of Twist and VEGF as well as high MVD values. In addition, it was indicated that Twist overexpression in OS may enhance cancer invasion and metastasis in OS through increasing VEGF expression, which in turn may result in increased MVD. Therefore, inhibition of Twist expression may have potential therapeutic use for the treatment of OS.

\section{Acknowledgements}

This study was supported by grants from the 2014 Hunan Provincial Innovation Foundation For Postgraduates (no. CX2014) and the Open-End Fund for the Valuable and Precision Instruments of Central South University (no. CSUZC2014046).

\section{References}

1. Yin K, Liao Q, He H and Zhong D: Prognostic value of Twist and E-cadherin in patients with osteosarcoma. Med Oncol 29: 3449-3455, 2012.

2. Mullen JT, Hornicek FJ, Harmon DC, et al: Prognostic significance of treatment-induced pathologic necrosis in extremity and truncal soft tissue sarcoma after neoadjuvant chemoradiotherapy. Cancer 120: 3676-3682, 2014.

3. Zhou Y, Zang X, Huang Z and Zhang C: TWIST interacts with endothelin-1/endothelin A receptor signaling in osteosarcoma cell survival against cisplatin. Oncol Lett 5: 857-861, 2013.

4. Levens D and Larson DR: A new twist on transcriptional bursting. Cell 158: 241-242, 2014.

5. Khan MA, Chen HC, Zhang D and Fu J: Twist: A molecular target in cancer therapeutics. Tumour Biol 34: 2497-2506, 2013.

6. Mironchik Y, Winnard PT Jr, Vesuna F, et al: Twist overexpression induces in vivo angiogenesis and correlates with chromosomal instability in breast cancer. Cancer Res 65: 10801-10809, 2005.

7. Niu RF, Zhang L, Xi GM, et al: Up-regulation of Twist induces angiogenesis and correlates with metastasis in hepatocellular carcinoma. J Exp Clin Cancer Res 26: 385-394, 2007.

8. Wallerand H, Robert G, Pasticier G, et al: The epithelial-mesenchymal transition-inducing factor TWIST is an attractive target in advanced and/or metastatic bladder and prostate cancers. Urol Oncol 28: 473-479, 2010.

9. Sossey-Alaoui K, Pluskota E, Davuluri G, et al: Kindlin-3 enhances breast cancer progression and metastasis by activating Twist-mediated angiogenesis. FASEB J 28: 2260-2271, 2014.

10. Low-Marchelli JM, Ardi VC, Vizcarra EA, van Rooijen N, Quigley JP and Yang J: Twist1 induces CCL2 and recruits macrophages to promote angiogenesis. Cancer Res 73: 662-671, 2013.
11. Tanaka T, Yui Y, Naka N, et al: Dynamic analysis of lung metastasis by mouse osteosarcoma LM8: VEGF is a candidate for anti-metastasis therapy. Clin Exp Metastasis 30: 369-379, 2013.

12. Sakurai T, Okumura H, Matsumoto M, et al: Endoglin (CD105) is a useful marker for evaluating microvessel density and predicting prognosis in esophageal squamous cell carcinoma. Anticancer Res 34: 3431-3438, 2014.

13. Wang WQ, Liu L, Xu HX, et al: The combination of HTATIP2 expression and microvessel density predicts converse survival of hepatocellular carcinoma with or without sorafenib. Oncotarget 5: 3895-3906, 2014

14. Ek ET, Ojaimi J, Kitagawa Y and Choong PF: Does the degree of intratumoural microvessel density and VEGF expression have prognostic significance in osteosarcoma? Oncol Rep 16: 17-23, 2006.

15. He S, Xiao Z, Chen L and Xiong S: Comment on, Xu XW, et al: Prognostic significance of VEGF expression in osteosarcoma: A meta-analysis. Tumour Biol 35: 6193-6194, 2014.

16. Becker RG, Galia CR, Morini $S$ and Viana CR: Immunohistochemical expression of vegf and her-2 proteins in osteosarcoma biopsies. Acta Ortop Bras 21: 233-238, 2013.

17. Ohba T, Cates JM, Cole HA, et al: Autocrine VEGF/VEGFR1 signaling in a subpopulation of cells associates with aggressive osteosarcoma. Mol Cancer Res 12: 1100-1111, 2014.

18. Roberts CC, Daffner RH, Weissman BN, et al: ACR appropriateness criteria on metastatic bone disease. J Am Coll Radiol 7: 400-409, 2010.

19. Zhou W, Hao M, Du X, Chen K, Wang G and Yang J: Advances in targeted therapy for osteosarcoma. Discov Med 17: 301-307, 2014.

20. Rettew AN, Getty PJ and Greenfield EM: Receptor tyrosine kinases in osteosarcoma: not just the usual suspects. Adv Exp Med Biol 804: 47-66, 2014.

21. Ohba K, Miyata Y, Matsuo T, et al: High expression of Twist is associated with tumor aggressiveness and poor prognosis in patients with renal cell carcinoma. Int J Clin Exp Pathol 7: 3158-3165, 2014.

22. Galvan JA, Helbling M, Koelzer VH, et al: TWIST1 and TWIST2 promoter methylation and protein expression in tumor stroma influence the epithelial-mesenchymal transition-like tumor budding phenotype in colorectal cancer. Oncotarget 6: 874-885, 2015.

23. Wang T, Li Y, Wang W, et al: Twist2, the key Twist isoform related to prognosis, promotes invasion of cervical cancer by inducing epithelial-mesenchymal transition and blocking senescence. Hum Pathol 45: 1839-1846, 2014.

24. Wang T, Li Y, Tuerhanjiang A, et al: Twist2 contributes to cisplatin-resistance of ovarian cancer through the AKT/GSK-3beta signaling pathway. Oncol Lett 7: 1102-1108, 2014.

25. Wang SW, Liu SC, Sun HL, et al: CCL5/CCR5 axis induces vascular endothelial growth factor-mediated tumor angiogenesis in human osteosarcoma microenvironment. Carcinogenesis 36 : 104-114, 2015.

26. Yu XW, Wu TY, Yi X, et al: Prognostic significance of VEGF expression in osteosarcoma: A meta-analysis. Tumour Biol 35: 155-160, 2014.

27. Zhou Q, Zhu Y, Deng Z, Long H, Zhang S and Chen X: VEGF and EMMPRIN expression correlates with survival of patients with osteosarcoma. Surg Oncol 20: 13-19, 2011.

28. Chen D, Zhang YJ, Zhu KW and Wang WC: A systematic review of vascular endothelial growth factor expression as a biomarker of prognosis in patients with osteosarcoma. Tumour Biol 34: 1895-1899, 2013.

29. Lu SM, Yu L, Tian JJ, et al: Twist modulates lymphangiogenesis and correlates with lymph node metastasis in supraglottic carcinoma. Chin Med J (Engl) 124: 1483-1487, 2011.

30. Che N, Zhao XL, Sun T, et al: The role of Twist1 in hepatocellular carcinoma angiogenesis: A clinical study. Hum Pathol 42: 840-847, 2011.

31. Dalal S, Berry AM, Cullinane CJ, et al: Vascular endothelial growth factor: A therapeutic target for tumors of the Ewing's sarcoma family. Clin Cancer Res 11: 2364-2378, 2005.

32. Kaya M, Wada T, Kawaguchi S, et al: Increased pre-therapeutic serum vascular endothelial growth factor in patients with early clinical relapse of osteosarcoma. Br J Cancer 86: 864-869, 2002

33. Oda Y, Yamamoto H, Tamiya S, et al: CXCR4 and VEGF expression in the primary site and the metastatic site of human osteosarcoma: Analysis within a group of patients, all of whom developed lung metastasis. Mod Pathol 19: 738-745, 2006. 